\title{
Distribución espacial de la probabilidad de ocurrencia de verdolaga silvestre (Portulaca oleracea L.) en la Región Lagunera de Coahuila, México
}

\author{
Spatial distribution of the probability of occurrence of wild purslane \\ (Portulaca oleracea L.) in the Region Lagunera of Coahuila, Mexico
}

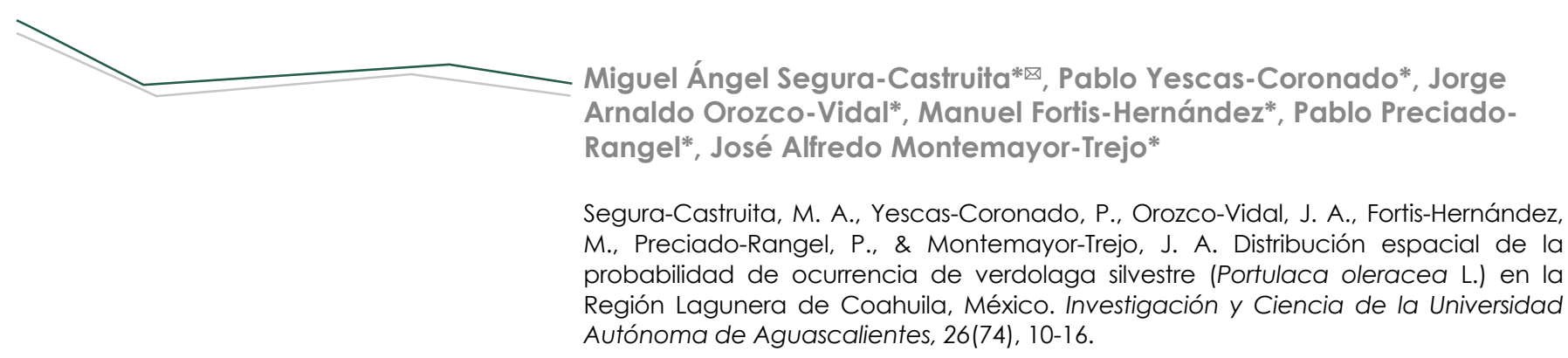

RESUMEN

El interés por introducir verdolaga (Portulaca oleracea L.) como planta cultivable se ha incrementado paulatinamente debido a sus propiedades medicinales. Sin embargo, estudios acerca de su distribución espacial y relación con el tipo de suelo son escasos. Los objetivos del presente trabajo fueron determinar la probabilidad de ocurrencia de verdolaga, así como la relación entre su distribución espacial y las características edáficas en la Región Lagunera. Se obtuvieron datos de presencia/ausencia de esta planta y muestras de suelo superficial de sitios en distintas clases de tierra, y se determinaron las características físicas y químicas de los suelos. Los datos obtenidos se utilizaron para estimar la probabilidad de ocurrencia de verdolaga con una regresión logística y se cartografiaron. Los resultados muestran que la arena, la conductividad eléctrica y el pH influyen en la presencia de la verdolaga y su distribución espacial es diferente, donde los suelos de tierras

Palabras clave: distribución espacial; interpolación Kriging; Portulaca oleracea; regresión logística; clases de tierras.

Keywords: spatial distribution; Kriging interpolation; land classes; logistic regression; Portulaca oleracea.

Recibido: 15 de agosto de 2017, aceptado: 7 de febrero de 2018

* División de Estudios de Posgrado e Investigación, Instituto Tecnológico de Torreón, Tecnológico Nacional de México. Antigua carretera Torreón-San Pedro km 7.5, ejido Ana, C. P. 27170, municipio de Torreón, Coahuila, México. Correo electrónico: dmilys5@hotmail.com pyescas@hotmail.com; joorvi66@hotmail.com; fortismanuel@hotmail. com; preciador@yahoo.com.mx; jtmontemayor@hotmail.com

$凶$

Autor para correspondencia blancas arenosas tuvieron la mayor probabilidad de ocurrencia.

\section{ABSTRAC1}

The interest in introducing purslane (Portulaca oleracea $\mathrm{L}$.) as a cultivable plant has been increased by its medicinal properties. However, studies of its spatial distribution and relation to the type of soil are scarce. The objectives of this work were to determine the probability of occurrence of purslane, and the relationship between its spatial distribution and the edaphic characteristics in the Region Lagunera. The presence/absence data of purslane and surface soil samples of sites in different land classes were obtained, and determined the physical and chemical characteristics of the soils. The data obtained were used to estimate the probability of occurrence of purslane with a logistic regression and were mapped. The results show that the sand, electrical conductivity and $\mathrm{pH}$ influence the presence of the purslane, being its spatial distribution different, where the soils of sandy white land had the greater probability of occurrence.

INTRODUCCIÓN

La verdolaga (Portulaca oleracea L.) es una especie de planta herbácea comestible, se distribuye en las regiones templadas y tropicales del mundo, y en América se conoce desde épocas precolombinas (Mera-Ovando, Bye-Boettler, \& Solano, 2014). Las hojas verdes de esta planta proporcionan antioxidantes, minerales, vitaminas y varios fitoquímicos con propiedades antioxidantes (Alam et al., 2015), entre ellos los ácidos grasos $\alpha$-linolénico y $\beta$-caroteno (Liu 


\section{IIVESTIGAGIÓn Y CUERCIA DE LA UחIVERSIDAD AUTÓNOMA DE RGUASCHLIETTES}

et al., 2000). P. oleracea destaca entre la mayoría de las especies de plantas silvestres comestibles por los escasos requerimientos para su desarrollo, de tal manera que en la actualidad existe un interés renovado por su cultivo (Palaniswamy, Bible, \& McAvoy, 2002).

En México, los estados de Baja California, Morelos, y la Ciudad de México cultivan verdolaga comercialmente, con una producción nacional de 6,000 Mg $\mathrm{ha}^{-1}$; lo anterior no significa que en el resto del país no crezca verdolaga, en las áreas de cultivo es parte del agrosistema. Aunque se le considera como indeseable o mala hierba, no lo es del todo, pues los agricultores la utilizan con fines alimenticios (Mera-Ovando et al., 2014). La amplia distribución de P. oleracea en todo el mundo se debe a su adaptabilidad a diferentes tipos de suelos y ambientes, particularmente a altas temperaturas, suelos salinos (Kafi \& Rahimi, 2011), condiciones de sequía (Ren et al., 2011), escasez nutrimental, además de su resistencia al frío (Rinaldi, Amodio, \& Colelli, 2010).

Por otra parte, es de considerarse que los productores poseen un conocimiento ancestral de sus tierras y que el concepto campesino de tierra, aunque cualitativo, es más amplio que el concepto científico de suelo (Ortiz-Solorio \& Gutiérrez-Castorena, 1999); el conocimiento que tienen de este último es detallado, ya que identifican características observables como color, retención de humedad y textura (Ortiz-Solorio, Gutiérrez-Castorena, Licona-Vargas, \& Sánchez-Guzmán, 2005); de esta manera, los campesinos o productores sistematizan este conocimiento en una clasificación de tierras (Licona-Vargas, Ortiz-Solorio, Gutiérrez-Castorena, \& Manzo-Ramos, 2006) y relacionan la presencia de diversas plantas silvestres con alguna clase de ellas (Ortiz-Solorio \& Gutiérrez-Castorena, 2001).

Las clases de tierra son cartografiables (BarreraBassols, Zinck, \& Van Ranst, 2006). Al respecto, SeguraCastruita et al. (2012) indican que los mapas de clases de tierra (MCT) pueden utilizarse como base para el análisis de suelos. Lo anterior permite deducir que la distribución espacial de verdolaga en una región árida se relacionaría con las características de los suelos de las clases de tierra que los campesinos de la región hayan identificado; no obstante, esta situación ha sido escasamente reportada. La delimitación de áreas potenciales para el desarrollo de plantas en función de las características de una región, si se tiene como base un MCT, es importante para la definición de planes de manejo (Licona-Vargas et al., 2006).
Uno de los esquemas metodológicos que se han empleado para establecer la distribución espacial de la probabilidad de ocurrencia de una especie de planta es la combinación del modelo de regresión logística (MRL) binaria y la cartografía asistida por computadora, a partir de datos de mapas de dependencias oficiales (Segura-Castruita et al., 2014). No obstante, la precisión podría incrementarse si los datos edáficos se obtienen de muestras de suelo recolectadas al momento de la verificación de la existencia de las plantas en campo. En función de lo anterior, si la obtención de las muestras de suelo y la verificación de una especie de planta (presencia o ausencia) se realizan en sitios de muestreo ubicados en forma aleatoria, basadas en un mapa de clases de tierra en una región árida, la precisión de los mapas de probabilidad de ocurrencia de la especie aumentaría.

Los objetivos del presente estudio fueron: (1) determinar la distribución espacial de la probabilidad de ocurrencia de P. oleracea y (2) cartografiar la distribución de la especie, tomado como base un mapa de clases de tierra de un área de cultivo de la Región Lagunera de Coahuila y verificar su precisión.

\section{MATERIALES Y MÉTODOS}

El área de estudio se localiza entre los $25^{\circ} 40^{\prime}-25^{\circ}$ $46^{\prime} \mathrm{N}$ y los $102^{\circ} 56^{\prime}-103^{\circ} 08^{\prime} \mathrm{O}$, con altitud promedio de 1,120 m, en una superficie de 75,155.49 ha, que corresponde a una parte de suelos cultivados del municipio de San Pedro, Coahuila (figura 1).

El clima es de tipo Bw(h')hw(e): seco desértico cálido con un régimen de lluvias en verano con oscilación extrema. La precipitación media anual es de $250 \mathrm{~mm}$, concentrada en los meses de junio a septiembre. Esta precipitación contrasta con la elevada evaporación media anual de 2,400 mm (García, 2004). El material parental está constituido por sedimentos aluviales del Cuaternario y se han identificado dos grupos de suelo (Calscisoles y Fluvisoles) a los que corresponden cuatro clases de tierras (SeguraCastruita et al., 2012).

El trabajo inició en el ciclo agrícola primaveraverano 2016 y concluyó en el de primavera 2017 , consistió en cinco etapas: 


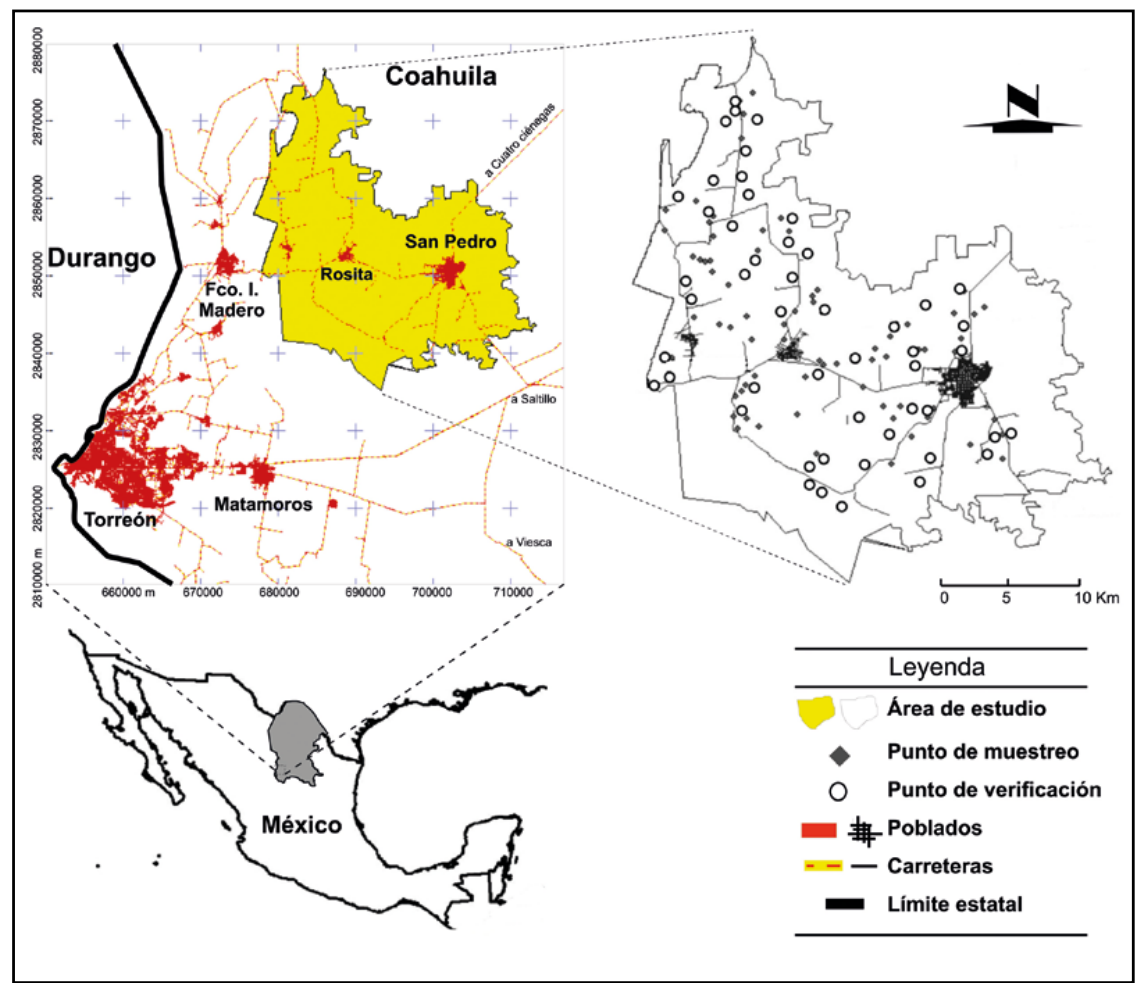

Figura 1. Localización del área de estudio.

Elaboración propia.

1) Selección de sitios de muestreo de plantas y suelos. Un mapa de clases de tierra (Segura-Castruita et al., 2012) del área de estudio se utilizó para ubicar sitios de muestreo. El tamaño de muestra (Schabenberger \& Pierce, 2002) fue de 72 sitios, distribuidos de forma aleatoria en cuatro clases de tierras [Blanca $(B /)$, Blanca arenosa $(B a)$, Prieta $(P r)$ y Negra (N)] que se utilizan para cultivo; cada clase tuvo 18 sitios de muestreo, se tuvo la precaución de que una unidad cartográfica de cada clase no tuviera más de un sitio; es decir, que se encontraran dispersos en la zona de estudio. En cada sitio se registró la ubicación geográfica, se verificó la existencia o ausencia de verdolaga y se colectó una muestra de suelo ( $2 \mathrm{~kg}$ aprox.) de la capa arable $(0-30 \mathrm{~cm}$ de profundidad), independientemente de la presencia o ausencia de la especie. Las muestras de verdolaga y de suelo se trasladaron a laboratorio.

2) Análisis de suelos. Las muestras de suelo se secaron a la sombra a temperatura ambiente, posteriormente se molieron y tamizaron. Las determinaciones físicas y químicas fueron: análisis mecánico de partículas (porcentajes de arena $[A]$, limo $[L]$ y arcilla $[R]$ ), densidad aparente (Da), $\mathrm{pH}$ y conductividad eléctrica (CE), características que se relacionan con el desarrollo de las plantas de verdolaga (Kafi \& Rahimi, 2011; Ren et al., 2011). Los análisis se realizaron siguiendo el Manual de Van Reeuwijk (2006).

3) Cartografía de la probabilidad de ocurrencia. Los datos de suelo estimados en una etapa anterior y la presencia o ausencia de Portulaca conformaron atributos que se utilizaron para generar una base de datos. Además, se estimaron los coeficientes de la regresión lineal múltiple (RLM) con el método de máxima verosimilitud, entre la variable dependiente (presencia de verdolaga) con las variables independientes (edáficas) y, posteriormente, con el método de regresión escalonada (RE), procedimiento de selección hacia adelante, se determinaron las variables con significancia estadística $(p \leq 0.05)$ en el desarrollo de verdolaga; es decir, se obtuvieron dos combinaciones lineales. Enseguida, la probabilidad de ocurrencia de verdolaga en cada punto de muestreo se obtuvo con el MRL binario de la ecuación (1), ya que permite asociar una variable dependiente con dos posibles valores (presencia o ausencia), con variables independientes (variables edáficas), utilizando la combinación lineal con mayor coeficiente de determinación $\left(R^{2}\right)$. 


\section{IIVESTIGAGIÓn Y CUERCIA DE LA UחIVERSIDAD AUTÓNOMA DE AGUASCALIERTES}

$$
p(v)=\frac{e^{z}}{\left(1+e^{z}\right)}
$$

donde $p(v)$ es la probabilidad de ocurrencia de verdolaga, $z$ es la combinación lineal de las diferentes variables en el estudio. La probabilidad de presencia de verdolaga en cada punto georreferenciado sirvió para actualizar la base de datos, siendo un atributo más. Los puntos georreferenciados y el atributo de probabilidad se utilizaron para realizar una interpolación con el modelo Inverse Distance Weighted (IDW) (Cruz-Cárdenas et al., 2010), con el software Arcgis 10.3.

4) Verificación de la precisión de los mapas. En cada clase de probabilidad se seleccionaron cinco sitios distribuidos aleatoriamente y se anotaron las coordenadas geográficas para posteriormente realizar la verificación en campo como lo indican Segura-Castruita et al. (2014), para lo cual se revisó solamente la presencia de plantas (sin considerar la abundancia o cantidad) en un área de $100 \mathrm{~m}^{2}(10 \mathrm{~m} \times 10 \mathrm{~m})$ alrededor del punto georreferenciado. El recorrido en campo se realizó un año después de la colecta, en el mismo periodo o mes con el fin de tener condiciones ambientales similares a las del muestreo. El análisis se realizó en un sentido discreto; es decir, acierto o error, se calculó el porcentaje de aciertos y se generó una matriz de precisión.

5) Análisis estadístico. Los resultados de las características del suelo y la presencia de verdolaga se sometieron a un análisis de varianza y se realizó una comparación de medias de Tukey ( $p \leq 0.05)$. Adicionalmente, los datos se relacionaron con las características de los suelos para determinar la presencia o ausencia de la especie con un MRL. Dichos procedimientos se realizaron en el programa Minitab 17.

\section{RESULTADOS}

Las clases de tierra tuvieron efectos significativos ( $p$ $\leq 0.05$ ) en la presencia de Portulaca oleracea L.; las tierras donde se encontró a esta especie fueron $B /$ y $\mathrm{Ba}$, principalmente. Los suelos de las cuatro clases mostraron diferencias significativas ( $p \leq 0.05)$ en los contenidos de A y R, Da y CE (tabla 1). Por otra parte, la presencia de plantas de verdolaga por clase, fue diferente (tabla 1). Además, en menos de la mitad de los sitios de muestreo se detectó esta especie de planta, su ocurrencia en función de las clases de tierra presentó el siguiente comportamiento: $\mathrm{Ba}>\mathrm{B} \mid>\mathrm{N}>\mathrm{Pr}$.

El análisis de regresión entre la presencia de verdolaga y las características de los suelos de las clases de tierra, indicó que A, CE y pH están asociadas a la ocurrencia de la especie ( $p \leq 0.05$, Tabla 2 ).

Por otra parte, la RLM de todas las variables con respecto a la presencia de verdolaga tuvo un valor de $R^{2}$ de 0.34 menor que la regresión escalonada $\left(R^{2}\right.$ $=0.7653$ ), lo cual indica que el segundo modelo es el más viable en el MRL.

La regresión logística dio como resultado diferentes probabilidades de ocurrencia para verdolaga, que variaron desde 6 hasta $88 \%$ (Tabla 3). No obstante, los valores de probabilidad no se distribuyeron uniformemente (figura 2).

Tabla 1

Características edáficas y frecuencias de presencia de las plantas de verdolaga en las clases de tierra

\begin{tabular}{ccccccccccc}
\hline $\begin{array}{c}\text { Clase } \\
\text { de tierra } \\
(\mathrm{CT})\end{array}$ & $\begin{array}{c}\mathrm{A}^{*} \\
(\%)\end{array}$ & $\begin{array}{c}\mathrm{L} \\
(\%)\end{array}$ & $\begin{array}{c}R \\
(\%)\end{array}$ & $\begin{array}{c}\mathrm{Da} \\
\left(\mathrm{g} \mathrm{cm}^{-3}\right)\end{array}$ & $\mathrm{pH}$ & $\begin{array}{c}\mathrm{CE} \\
\left(\mathrm{dS} \mathrm{m} \mathrm{m}^{-1}\right)\end{array}$ & $\mathrm{N}$ & $P$ & $A$ & $\begin{array}{c}F O \\
(\%)\end{array}$ \\
\hline $\mathrm{Pr}$ & $22 \mathrm{c}$ & $40 \mathrm{c}$ & $38 \mathrm{a}$ & $1.35 \mathrm{~b}$ & $7.6 \mathrm{c}$ & $0.59 \mathrm{~b}$ & 18 & 4 & 14 & 22.2 \\
$\mathrm{~N}$ & $29 \mathrm{a}^{* *}$ & $38 \mathrm{~d}$ & $33 \mathrm{~b}$ & $1.36 \mathrm{~b}$ & $7.9 \mathrm{~b}$ & $0.72 \mathrm{~b}$ & 18 & 5 & 13 & 27.7 \\
$\mathrm{Bl}$ & $9 \mathrm{~d}$ & $62 \mathrm{a}$ & $29 \mathrm{c}$ & $1.36 \mathrm{~b}$ & $7.6 \mathrm{c}$ & $0.62 \mathrm{~b}$ & 18 & 10 & 8 & 55.5 \\
$\mathrm{Ba}$ & $24 \mathrm{~b}$ & $54 \mathrm{~b}$ & $22 \mathrm{~d}$ & $1.48 \mathrm{a}$ & $8.2 \mathrm{a}$ & $4.38 \mathrm{a}$ & 18 & 15 & 3 & 83.3 \\
$\mathrm{DSH}$ & 0.09 & 1.97 & 1.99 & 0.03 & 0.19 & 0.32 & & & & \\
Total & & & & & & & 72 & 34 & 38 & 47.3 \\
\hline
\end{tabular}

Nota: "A: arena, L: Limo, R: arcilla, Da: densidad aparente, CE: conductividad eléctrica, CT: clase de tierra. N: tamaño de muestra, P: presencia, A: ausencia, FO: frecuencia de ocurrencia.

**Letras distintas en la misma columna reflejan diferencias estadísticas significativas (Tukey, p $\leq 0.05$ ), DHS: Diferencia significativa honesta ( $p \leq 0.05$ ).

Elaboración propia. 


\section{IIVESTIGAGIÓก Y CIECEIA DE LA UNIVERSIDAD AUTÓNOMA

Tabla 2

Coeficientes de regresión de la presencia de verdolaga en función de las características edáficas

\begin{tabular}{ccccccc}
\hline \multirow{2}{*}{ Variable } & \multicolumn{3}{c}{$R L M$} & & \multicolumn{3}{c}{$R E$} \\
\cline { 2 - 7 } & $\beta^{*}$ & $E$ & $P$ & $\beta$ & $E$ & $P$ \\
\hline Intercepto & -3.520 & 8.540 & & -29.100 & 13.200 & \\
$A(\%)$ & -0.066 & 0.029 & 0.023 & -0.105 & 0.046 & 0.015 \\
$L(\%)$ & -0.045 & 0.025 & 0.081 & - & - & - \\
$R(\%)$ & -0.032 & 0.024 & 0.187 & - & - & - \\
$D a\left(\mathrm{~g} \mathrm{~cm}^{-3}\right)$ & 0.320 & 1.210 & 0.790 & - & - & - \\
$\mathrm{pH}$ & 0.545 & 0.217 & 0.014 & 3.84 & 1.78 & 0.019 \\
$C E\left(\mathrm{dS} \mathrm{m}^{-1}\right)$ & 0.157 & 0.063 & 0.016 & 0.54 & 0.29 & 0.045 \\
\hline
\end{tabular}

Nota: " $\beta$ : coeficiente de regresión, E: error, P: probabilidad de rechazo $(p \leq 0.05)$,

RLM: regresión lineal múltiple, RE: regresión escalonada.

Elaboración propia.

Tabla 3

Clases de probabilidad de ocurrencia de P. oleracea, área que ocupan y matriz de validación del mapa de ocurrencia

\begin{tabular}{|c|c|c|c|c|c|}
\hline $\begin{array}{c}\text { Clase de Probabilidad } \\
(\%)\end{array}$ & $\begin{array}{l}\text { Área } \\
\text { (ha) }\end{array}$ & $N^{*}$ & Acierto & Error & $\begin{array}{c}\text { Precisión } \\
(\%)\end{array}$ \\
\hline $0-10$ & 1678.88 & 5 & 2 & 3 & 40 \\
\hline $10-20$ & 10508.13 & 5 & 3 & 2 & 60 \\
\hline $20-30$ & 14033.16 & 5 & 3 & 2 & 60 \\
\hline $30-40$ & 19555.65 & 5 & 4 & 1 & 80 \\
\hline $40-50$ & 12643.11 & 5 & 4 & 1 & 80 \\
\hline $50-60$ & 7466.58 & 5 & 4 & 1 & 80 \\
\hline $60-70$ & 3677.94 & 5 & 5 & - & 100 \\
\hline $70-80$ & 2736.81 & 5 & 5 & - & 100 \\
\hline $80-90$ & 2880.18 & 5 & 5 & - & 100 \\
\hline $90-100$ & 995.04 & 5 & 5 & - & 100 \\
\hline Total & 75155.49 & 50 & 40 & 10 & 80 \\
\hline
\end{tabular}

Nota: "N: número de muestras.

Elaboración propia.

DISCUSIÓN

Los suelos de las clases $\mathrm{Ba}$ y $\mathrm{Bl}$, donde se distribuye principalmente $P$. oleracea, se han clasificado dentro de la subunidad Calcisoles hapli-arídicos. En cambio, las $\operatorname{Pr}$ y $\mathrm{N}$ se clasifican dentro de las subunidades Fluvisoles hapli-áridicos y Fluvisoles calci-arídicos, respectivamente (Segura-Castruita et al., 2012), suelos que poseen características contrastantes. Los Calcisoles se caracterizan por el alto contenido de carbonato de calcio $\left(\mathrm{CaCO}_{3}\right)$ secundario, por efecto de las condiciones áridas; mientras que los Fluvisoles tienen materiales flúvicos; es decir, formados por sedimentos fluviales que reciben material recien- te o lo han recibido en el pasado y todavía muestran estratificación (IUSS Working Group WRB, 2015). Al respecto, Brady y Weil (2008) mencionan que las características físicas y químicas de los suelos influyen en la presencia o ausencia de las plantas. En el presente estudio se encontró que la relación entre la textura (por el incremento en el contenido de arcilla) y la presencia de la verdolaga fue significativa; mientras que el alto contenido de arena también favoreció su presencia, como lo mencionan Blázquez y Carbó (2015). La presencia de Portulaca oleracea se ha registrado en sitios con $\mathrm{pH}$ desde ácidos hasta alcalinos y en $\mathrm{pH}$ desde neutros hasta ligeramente alcalinos, como en zonas áridas (Kafi \& Rahimi, 2011). 


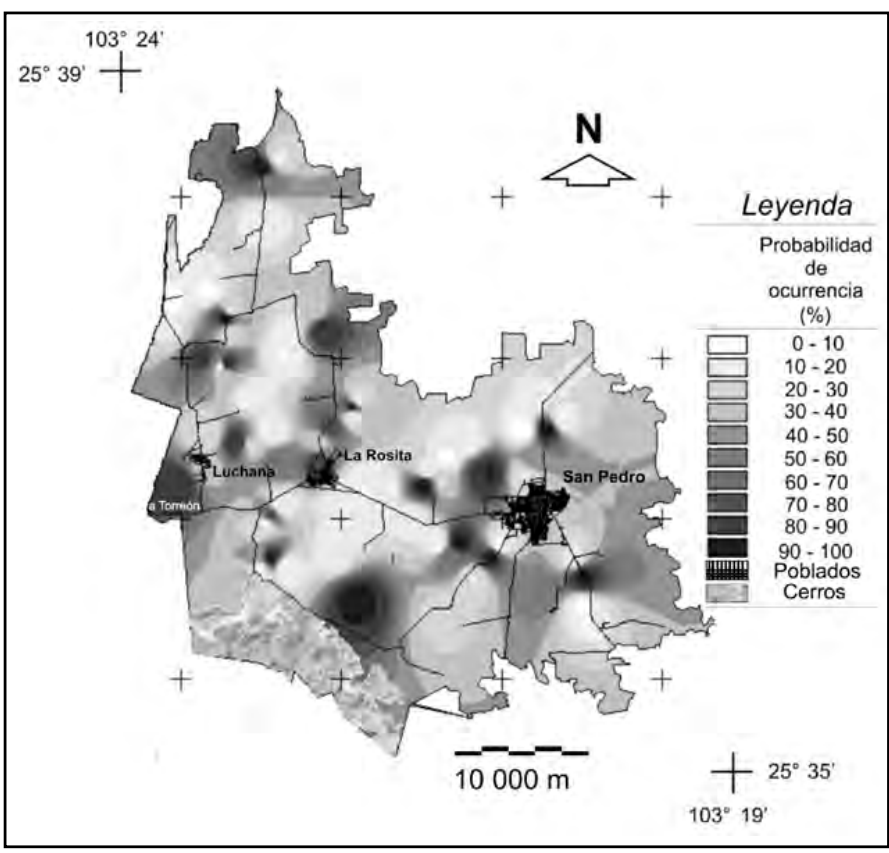

Figura 2. Mapa de probabilidades de ocurrencia de $P$. oleracea en San Pedro, Coah., México.

Elaboración propia.

Por otra parte, la verdolaga es tolerante a la salinidad; así que se desarrolla en ambientes no salinos (< $2 \mathrm{dsm}^{-1}$ ) o CE entre 2 y $6.8 \mathrm{dsm}^{-1}$, que se consideran como moderadamente salinos (Teixeira \& Carvalho, 2009).

Los resultados anteriores explican por qué las características edáficas del área de estudio influyeron de manera diferente en la distribución espacial de la probabilidad de ocurrencia de la verdolaga; aunque en el modelo lineal el signo de los coeficientes de las tres variables que influyeron en la presencia de la especie fueron diferentes. La arena tuvo un coeficiente con signo negativo, mientras que en la $\mathrm{CE}$ y el $\mathrm{pH}$ el mismo fue positivo. En este sentido, el signo de los coeficientes indica el incremento o decremento de la probabilidad de ocurrencia de la especie vegetal; es decir, cuando el signo es negativo, al incrementarse la variable independiente la probabilidad de ocurrencia disminuye; en cambio, con el signo positivo, al aumentar la variable, la probabilidad se incrementa (Segura-Castruita et al., 2014). Lo anterior sugiere que la verdolaga puede encontrarse en cualquier suelo agrícola de San Pedro, y se puede deducir que el incremento en el porcentaje de arena más allá del promedio de las tierras Ba y $B I$ podría disminuir la probabilidad de ocurrencia de $P$. oleracea, mientras que el aumento de la CE y el pH la incrementarían. Esta especie de planta responde a condiciones de estrés hídrico y por salinidad con un mecanismo de defensa enzimático que produce bioactivos; por tanto, esta especie tolera incrementos en la salinidad del suelo mayores que $6.8 \mathrm{dS} \mathrm{m}^{-1}$ (Alam et al., 2015; Yazici, Türkan, Sekmen, \& Demiral, 2007); situación que predijeron los coeficientes positivos de las variables $\mathrm{CE}$ y $\mathrm{pH}$ en el modelo de regresión logística, lo que indica la validez de este modelo.

La precisión del mapa de probabilidad de ocurrencia de verdolaga fue ligeramente superior al de otros mapas predictivos (76.2\%) validados con información de campo (Segura-Castruita et al., 2014). Al respecto, van Horssen, Pebesma y Schot (2002) mencionan que la calidad y cobertura de datos espaciales pueden afectar el resultado de la cartografía, situación que explicaría el incremento de la precisión en el presente estudio, ya que los datos edáficos se obtuvieron de muestras de suelo que se tomaron en campo al momento de la verificación de presencia de la planta.

\section{CONCLUSIONES}

La utilización de mapas de clases de tierras campesinas para la ubicación de sitios para la verificación de la ocurrencia de Portulaca oleracea y el muestreo de suelo permitieron determinar que la presencia de esta planta se relaciona con el porcentaje de arena, $\mathrm{pH}$ y CE de los suelos agrícolas en una región árida. Al utilizar una función lineal múltiple con dichas características (variables independientes) y la presencia de verdolaga (variable dependiente), en el modelo de regresión logística, se obtiene la estimación de la probabilidad de ocurrencia de esta planta con una precisión cartográfica de $80 \%$. Aun cuando no se verificó la calidad fitoquímica de la verdolaga silvestre en los suelos analizados, se requiere de mayor investigación para verificar si la distribución espacial de P. oleracea está relacionada con su capacidad antioxidante y contenido de ácidos grasos.

Por otra parte, el esquema metodológico que se presentó en este trabajo es una alternativa aplicable para la definición de áreas de ocurrencia de una especie de planta en función de las características edáficas de lugares donde las características climáticas permanecen como constantes. Asimismo, los resultados presentan áreas potenciales para el cultivo de P. oleracea en la Región Lagunera. 
- Alam, M. A., Juraimi, A. S., Rafi, M. Y., Hamid, A. A., Aslani, F. \& Alam, M. Z. (2015). Effects of salinity and salinity-induced augmented bioactive compounds in purslane (Portulaca oleracea L.) for possible economical use. Food Chemistry, 169, 439-447.

- Barrera-Bassols, N., Zinck, J. A., \& Van Ranst, E. (2006). Local soil classification and comparison of indigenous and technical soil maps in Mesoamerican community using spatial analysis. Geoderma, 135, 140-162.

- Blázquez, M. A., \& Carbó, E. (2015). Control of Portulaca oleracea by boldo and lemon essential oils in different soils. Industrial Crops and Products, 76, 515-521.

- Brady, N. C., \& Weil, R. R. (2008). The nature and properties of soils. (14th. ed.). Upper Saddle River, NJ, US: Pearson-Prentice Hall.

- Cruz-Cárdenas, G., Ortiz-Solorio, C. A., Ojeda-Trejo, E., Martínez-Montoya, J. F., Sotelo-Ruiz, E. D., \& Licona-Vargas, A. L. (2010). Evaluation of four digital classifiers for automated cartography of local soil classes based on reflectance and elevation in Mexico. International Journal of Remote Sensing, 31 (3), 665-679.

- García, E. (2004). Modificaciones al sistema de clasificación climática de Köppen. D. F., México: Universidad Nacional Autónoma de México.

- IUSS Working Group WRB. (2015). World Reference Base for Soil Resources 2014: International soil classification system for naming soils and creating legends for soil maps. World Soil Resources Reports No. 106. Rome, Italy: FAO.

- Kafi, M., \& Rahimi, Z. (2011). Effect of salinity and silicon on root characteristics, growth, water status, proline content and ion accumulation of purslane (Portulaca oleracea L.). Soil Science and Plant Nutrition, 57(2), 341-347.

- Licona-Vargas, A. L., Ortiz-Solorio, C. A., Gutiérrez-Castorena, M. C., \& Manzo-Ramos, F. (2006). Clasificación local de tierras y tecnología del policultivo café-plátano para velillo-sombra en comunidades cafetaleras. Terra Latinoamericana, 24(1), 1-7.

- Liu, L., Howe, P., Zhou, Y. F., Xu, Z. Q., Hocart, C., \& Zhan, R. (2000). Fatty acids and beta-carotene in australian purslane (Portulaca oleracea) varieties. Journal of Chromatography A, 893(1), 207-213.

- Mera-Ovando, L. M., Bye-Boettler, A., \& Solano, M. L. (2014). La verdolaga (Portulaca oleracea L.). Fuente natural de Omega 3 y Omega 6. Agroproductividad, 7(1), 3-7.

- Ortiz-Solorio, C. A., \& Gutiérrez-Castorena, M. C. (1999). Evaluación taxonómica de sistemas locales de clasificación de tierras. Terra Latinoamericana, 17(4), 277-286.
trospectiva. Etnobiología, 1 (1), 44-62.

- Ortiz-Solorio, C. A., Gutiérrez-Castorena, M. C., Licona-Vargas, A. L., \& Sánchez-Guzmán, P. (2005). Contemporary influence of indigenous soil (Land) classification in Mexico. Eurasian Soil Science, 38, S89-594.

- Palaniswamy, U. R., Bible, B. B., \& McAvoy, R. J. (2002). Effect of Nitrate: Ammonium Nitrogen Ratio on Oxalate Levels of Purslane. En J. Janick \& A. Whipkey (Eds.), Trends in new crops and new uses (pp. 453-455). Alexandria, VA: ASHS Press.

- Ren, S., Weeda, S., Akande, O., Guo, Y., Rutto, L., \& Mebrahtu, T. (2011). Drought tolerance and AFLP-based genetic diversity in purslane (Portulaca oleracea L.). Journal of Biotech Research, 3(1), 51-61.

- Rinaldi, R., Amodio, M. L., \& Colelli, G. (2010). Effect of temperature and exogenous ethylene on the physiological and quality traits of purslane (Portulaca oleracea L.) leaves during storage. Postharvest Biology and Technology, 58(2), 147-156.

- Schabenberger, O., \& Pierce, F. J. (2002). Contemporary statistical models for the plants and soil science. Boca Raton, FL: CRC Press LLC.

- Segura-Castruita, M. A., Huerta-García, A., Fortis-Hernández, M., Montemayor-Trejo, J. A., Martínez-Corral, L., \& YescasCoronado, P. (2014). Cartografía de la probabilidad de ocurrencia de Atriplex canescens en una región árida de México. Agrociencia. 48(6): 639-652.

- Segura-Castruita, M. A., Martínez-Corral, L., García-Barrientos, E., Huerta-García, A., García-Hernández, J. L., Fortis-Hernández, M..... Preciado-Rangel, P. (2012). Localization of local soil classes in an arid region of Mexico, using satellite imagery. International Journal of Remote Sensing, 33(1), 184-197.

- Teixeira, M., \& Carvalho, I. S. (2009). Effects of salt stress on purslane (Portulaca oleracea) nutrition. Annals of Applied Biology, $154(1), 77-86$.

- Van Horssen, P. W., Pebesma, E. J., \& Schot, P. P. (2002). Uncertainties in spatially aggregated predictions from a logistic regression model. Ecological Modelling, 154(1-2), 93-101.

- Van Reeuwijk, L. P. (2006). Procedures for soil analysis (7th. ed.). Tech. Pap. 9. Wageningen, Netherlands: ISRIC-World Soil Information.

- Yazici, I., Türkan, I., Sekmen, A. H., \& Demiral, T. (2007). Salinity tolerance of purslane (Portulaca oleracea L.) is achieved by enhanced antioxidative system, lower levels lipid peroxidation and proline accumulation. Environmental and Experimental Botany, 61 (1), 49-57. 